\title{
SUMS OF POWERS OF CONJUGATES OF ALGEBRAIC NUMBERS
}

\author{
P. E. BLANKSBY
}

ABSTRACT. This note is primarily concerned with algebraic numbers which have at least one conjugate with modulus exceeding one. Theorems which give lower bounds for the maximum of the moduli of the conjugates of such algebraic numbers are connected with equivalent theorems giving lower bounds for the sums of powers of the conjugates. The results relate to some earlier work of S. Chowla, and the method used depends on one of P. Turán's main theorems on lower bounds for sums of powers of complex numbers.

1. Throughout this paper we will assume that $\alpha$ is an algebraic number of degree $n(n>1)$ with conjugates $\alpha=\alpha_{1}, \alpha_{2}, \ldots, \alpha_{n}$. If $\alpha$ is a zero of an irreducible, primitive polynomial over the rational integers, with positive leading coefficient $d$, then we will call $d$ the denominator of $\alpha^{1}$

We have the following basic fact. For each pair of positive integers $n$ and $d$, there exists a positive real number $\phi(n, d)$, with the property that for algebraic numbers $a$ of degree $n$ and denominator $d$,

$$
\begin{array}{rlrl}
\text { either } & & \leq 1, \\
\text { or } & & & \geq 1+1 / \phi(n, d),
\end{array}
$$

where $|a|=\max _{1 \leq k \leq n}\left|\alpha_{k}\right|$. This follows since bounds on $|a|$ provide bounds on the elementary symmetric functions of $\alpha_{1}, \alpha_{2}, \ldots, a_{n}$, which in turn provide bounds (in terms of $n$ and $d$ ) on the coefficients of possible defining polynomials for such $a$. The problem of finding the best possible function $\phi$ with this property is not investigated here; it is proposed to pursue this else-

Received by the editors March 8, 1974.

AMS (MOS) subject classifications (1970). Primary 12D10, 30 A08; Secondary $10 \mathrm{~F} 10,12 \mathrm{~A} 15$.

Key words and phrases. Algebraic numbers, conjugates.

${ }^{1}$ This usage is only a matter of convenience here; clearly $d$ need not be the "least denominator" of $a$ in the obvious sense. In fact, if $D$ is the least of the positive integers $D^{\prime}$ such that $D^{\prime}$ a is an algebraic integer, then $D \leq d \leq D^{n}$. A result analogous to our Theorem 3 that follows then holds, with $\phi$ replaced by a corresponding function $\Phi=\Phi(n, D)$. 
where. It has been shown [1] that $\phi(n, 1)=30 n^{2} \log 6 n$ is valid in (1).

We define, for integral $k$,

$$
s_{k}(\alpha)=\sum_{j=1}^{n} \alpha_{j}^{k} .
$$

S. Chowla [2] has proved the following theorem involving $S_{k}(\alpha)$.

Theorem (Chowla). Let $a_{n} x^{n}+a_{n-1} x^{n-1}+\cdots+a_{0}$ be a reciprocal poly. nomial of degree $n$ with integral coefficients, and with zeros $\alpha_{1}, \alpha_{2}, \ldots, a_{n}$. If $\left|s_{k}(\alpha)\right| \leq n$ for all $k$ satisfying $0 \leq k \leq H^{c n^{2}}$, where $H=n+\sum_{j=0}^{n}\left|a_{j}\right|$, and $c$ is a positive constant independent of $n$ and $H$, then $\left|\alpha_{j}\right|=1$ for $1 \leq j \leq n$.

In this note we will see that results of the type (1) are connected with results related to Chowla's. We prove in the case $d=1$

Theorem 1. For every algebraic integet a of degree $n(n>1)$ which is not a root of unity, there exists an integer $k$ satisfying $1 \leq k \leq 2000 n^{3}(\log 6 n)^{2}$ and such that $\left|S_{k}(\alpha)\right|>\left(30 n^{3} \log 6 n\right)^{n+1}$.

There is no special significance in the numerical constant 2000, which could be reduced in size by more detailed calculations.

2. Before proceeding to the proof of Theorem 1, we make the following observations about algebraic numbers $a$.

(i) If $\alpha$ is reciprocal (that is $\alpha^{-1}$ is a conjugate of $\alpha$ ), as in Chowla's result, then the condition $|a| \leq 1$ implies that $\left|a_{k}\right|=1$ for all $1 \leq k \leq n$.

(ii) If $\left|a_{j}\right|=1$ for some $1 \leq j \leq n$, then $\alpha$ is reciprocal. In particular, if $a=1$, then $a$ is reciprocal and so $\left|\alpha_{k}\right|=1$ for all $1 \leq k \leq n$. In fact, it is easy to see that $\left|a_{k}\right|=|a|$ for all $1 \leq k \leq n$, if and only if some positive integral power of $a$ is a rational number.

(iii) In the case when $d=1$ (that is $\alpha$ is an algebraic integer) and $a \leq 1$, then a classical theorem of Kronecker [3] implies that $\alpha$ is a root of unity.

(iv) For algebraic numbers $a$, the superior limit of $\left|S_{k}(a)\right|$ as $k \rightarrow \infty$ is clearly determined by the size of $a$. In fact $\lim _{\sup _{k \rightarrow \infty}}\left|s_{k}(\alpha)\right|$ equals $0, n$, or is infinite, according as to whether $|a|<1,|a|=1$, or $|a|>1$, respectively. For, if $\epsilon$ is arbitrary, satisfying $0<\epsilon<1$, we can apply Dirichlet's theorem on simultaneous approximations to the $n$ real numbers $(1 / 2 \pi)$ arg $\alpha_{j}$ to determine infinitely many positive integers $k$ such that the corresponding complex numbers $\alpha_{1}^{k}, \alpha_{2}^{k}, \ldots, a_{n}^{k}$ all lie in the sector defined by $|\theta|<\operatorname{arc} \cos (1-\epsilon)$. 
For such $k$,

$$
\left|S_{k}(\alpha)\right| \geq \operatorname{Re} S_{k}(\alpha)=\sum_{j=1}^{n} \operatorname{Re} \alpha_{j}^{k}>(1-\epsilon) \sum_{j=1}^{n}\left|\alpha_{j}^{k}\right| .
$$

Thus for these $k$ we have, in general,

$$
\left|s_{k}(\alpha)\right|>(1-\epsilon)|\propto|^{k},
$$

and when $|a|=1$ (and so $\left|\alpha_{k}\right|=1$ for all $1 \leq k \leq n$ by (ii)), $\left|S_{k}(\alpha)\right|>(1-\epsilon) n_{\text {。 }}$ The above claims follow from these inequalities.

3. Proof of Theorem 1. The theorem is an immediate consequence of the following result for complex numbers.

Theorem 2. For every integer $n(n>1)$ and every real number $\phi(\phi \geq 1)$, the following two statements hold for all lists of $n$ complex numbers $z_{1}, z_{2}$, $\cdots, z_{n}$ :

(i) the inequality

$$
\max _{1 \leq k \leq n \phi \log (n \phi)}\left|z_{1}^{k}+z_{2}^{k}+\cdots+z_{n}^{k}\right|>(n \phi)^{n+1}
$$

implies the inequality

$$
\max _{1 \leq k \leq n}\left|z_{k}\right|>1+\phi^{-1}
$$

(ii) the inequality

$$
\max _{1 \leq k \leq n}\left|z_{k}\right| \geq 1+\phi^{-1}
$$

implies the inequality

$$
\max _{1 \leq k \leq 20 n \phi \log (n \phi)}\left|z_{1}^{k}+z_{2}^{k}+\cdots+z_{n}^{k}\right|>(n \phi)^{n+1} .
$$

If, as in the hypothesis of Theorem 1, $\alpha$ is an algebraic integer which is not a root of unity, then $a>1$, and in fact, by the result already mentioned [1], $|\propto|>1+\left(30 n^{2} \log 6 n\right)^{-1}$, where $n$ is the degree of $a$. Thus on taking $z_{k}=a_{k}(1 \leq k \leq n)$, and $\phi=\phi(n)=30 n^{2} \log 6 n$ in Theorem 2(ii), the result of Theorem 1 follows directly, since $30 n^{3} \log 6 n<(6 n)^{10 / 3}$.

Of course, Theorem 2 applies equally well to the general case of algebraic numbers with denominator $d>1$, and so provides an equivalence between results of the type (1) of the second paragraph and results similar to that of Chowla. For example, the following theorem is also a direct application of Theorem 2 .

Theorem 3. If $\phi=\phi(n, d)$ is any function which is valid for the basic 
result (1), then for every algebraic number a of degree $n$ and denominator $d$ for which $|a|>1$, there exists an integer $k$ satisfying $1 \leq k \leq 20 n \phi(n, d)$. $\log (n \phi(n, d))$ and such that $\left|S_{k}(\alpha)\right|>(n \phi(n, d))^{n+1}$.

4. Proof of Theorem 2. (i) This follows after using the triangle inequality.

(ii) We follow Chowla's method and apply Turán's second main theorem [4, Theorem IX]. The following more recent version of this inequality may be found in van der Poorten [5, Corollary to Theorem 1]:

If $z_{1}, z_{2}, \cdots, z_{n}$ and $a_{1}, a_{2}, \cdots, a_{n}$ denote complex numbers, and $\left|z_{1}\right| \geq\left|z_{j}\right|(1 \leq j \leq n)$, then for any integer $m \geq-1$ there exists an integer $k$ satisfying $m+1 \leq k \leq m+n$, and

$$
\sum_{j=1}^{n} a_{j} z_{j}^{k} \geq \frac{1}{8}\left(\frac{n-1}{8 e(n+m)}\right)^{n-1} \min _{1 \leq j \leq n}\left|a_{1}+\cdots+a_{j}\right|\left|z_{1}\right|^{k} .
$$

For notational convenience we write

$$
M=\max _{1 \leq k \leq n}\left|z_{k}\right| \text { and } S_{k}=\left|z_{1}^{k}+z_{2}^{k}+\cdots+z_{n}^{k}\right|
$$

By Turán's result, there exists an integer $k$ with $m+1 \leq k \leq m+n$ and

$$
S_{k} \geq \frac{M^{k}}{8}\left(\frac{n-1}{8 e(n+m)}\right)^{n-1}
$$

Assuming $n \geq 2, m \geq 1$, and $M>1$ we get

$$
S_{k} \geq \frac{1}{8} M^{m+1}(8 e(m+2))^{1-n} \geq \frac{1}{8} M^{m+1}(24 e m)^{1-n} \geq M^{m+1}(24 e m)^{-n} \text {. }
$$

If $[x]$ denotes the integer part of $x$, define $m=[18 n \phi \log (n \phi)]$. Using the inequalities $\left(1+\phi^{-1}\right)^{\phi} \geq 2$ and $e \log x \leq x$, we derive

$$
\begin{aligned}
S_{k}^{1 / n} & \geq\left(1+\phi^{-1}\right)^{18 \phi \log (n \phi)}(432 e n \phi \log (n \phi))^{-1} \\
& \geq 2^{18 \log (n \phi)}\left(432(n \phi)^{2}\right)^{-1} \\
& =\frac{1}{432}(n \phi)^{18 \log 2-2}>(n \phi)^{3 / 2} \geq(n \phi)^{(n+1) / n}
\end{aligned}
$$

Thus we have $S_{k}>(n \phi)^{n+1}$ for some $k$ satisfying

$$
18 n \phi \log (n \phi) \leq k \leq 18 n \phi \log (n \phi)+n<20 n \phi \log (n \phi),
$$

which concludes the proof of (ii). 


\title{
REFERENCES
}

1. P. E. Blanksby and H. L. Montgomery, Algebraic integers near the unit circle, Acta Arith. 18 (1971), 355-369. MR 45\#5082.

2. S. Chowla, On polynomials all of whose roots lie on the unit circle, J. Reine Angew. Math. 222 (1966), 69-70. MR 32 \#5604.

3. L. Kronecker, Zwei Sätze über Gleichungen mit ganzzahligen Coefficienten, J. Reine Angew. Math. 53 (1857), 173-175.

4. Paul Turán, Eine neue Methode in der Analysis und deren Anwendungen, Akad. Kiadó, Budapest, 1953. MR 15, 688.

5. A. J. van der Poorten, Generalisations of Turán's main theorems on lower bounds for sums of powers, Bull. Austral. Math. Soc. 2(1970), 15-37. MR 42 \#217.

DEPARTMENT OF PURE MATHEMATICS, UNIVERSITY OF ADELAIDE, ADELAIDE, SOUTH AUSTRALIA, AUSTRALIA 5001

PROCEEDINGS OF THE

AMERICAN MATHEMATICAL SOCIETY

Volume 49, Number 1, May 1975

\section{THE DIMENSION OF THE RING OF COEFFICIENTS IN A POLYNOMIAL RING}

\author{
JIMMY T. ARNOLD
}

ABSTRACT. $A$ and $B$ are commutative rings with identity. We say that $A$ and $B$ are stably equivalent provided there exists a positive integer $n$ such that the polynomial rings $A\left[X_{1}, \cdots, X_{n}\right]$ and $B\left[Y_{1}, \cdots, Y_{n}\right]$ are isomorphic. If $A$ and $B$ are stably equivalent, then they have equal Krull dimen sion.

The question answered in this paper arises from recent investigations concerning the uniqueness of the ring of coefficients in a polynomial ring (cf. [1]-[6]). In [6], Hochster has given an example which illustrates that stably equivalent rings need not be isomorphic. Several related questions are posed by Eakin and Heinzer in [5]. In particular, if $A$ and $B$ are stably equivalent rings, then Eakin and Heinzer ask whether $\operatorname{dim} A=\operatorname{dim} B(\operatorname{dim} R$ denotes the Krull dimension of the ring $R$ ). We shall presently show that this

Received by the editors February 1, 1974.

AMS (MOS) subject classifications (1970). Primary 13B25; Secondary 13A15, $13 \mathrm{C} 15$.

Key words and phrases. Polynomial ring, Krull dimension, coefficient ring. 